Kragujevac Journal of Mathematics

Volume 41(1) (2017), Pages 121-141.

\title{
EXPLICIT AND RECURSIVE FORMULAS, INTEGRAL REPRESENTATIONS, AND PROPERTIES OF THE LARGE SCHRÖDER NUMBERS
}

\author{
FENG QI ${ }^{1,2,3}$ AND BAI-NI GUO ${ }^{4}$
}

\begin{abstract}
In the paper, the authors review and survey some new results on the large Schröder numbers. These results were obtained by the authors and their coauthors from January 2016 and can be summarized up as follows.

(a) By studying the generating function of the large Schröder numbers, the authors and their coauthors establish several explicit formulas and a recursive formula for the large Schröder numbers and present relations between the Schröder numbers and central Delannoy numbers.

(b) By utilizing the Cauchy integral formula in the theory of complex functions, the authors and their coauthors build several integral representations for the large Schröder numbers and their generating function.

(c) By employing integral representations for the large Schröder numbers, the authors and their coauthors find some properties, including the convexity, complete monotonicity, product inequalities, and determinantal inequalities, of the large Schröder numbers.
\end{abstract}

\section{What ARE The SChrÖDER Numbers?}

Let us recall some basic knowledge about the Schröder numbers.

1.1. What are the Schröder Numbers? In combinatorics and number theory, there are two kinds of Schröder numbers, the large Schröder numbers $S_{n}$ and the

Key words and phrases. Large Schröder number, little Schröder number, generating function, explicit formula, recursive formula, integral representation, convexity, logarithmic convexity, complete monotonicity, determinantal inequality, product inequality, central Delannoy numbers, relation.

2010 Mathematics Subject Classification. Primary: 11B83. Secondary: 05A15, 11R33, 11Y55, 26A48, 30E20, 33B99, 44A10, 44A15.

Received: June 12, 2016.

Accepted: August 19, 2016. 
little Schröder numbers $s_{n}$. They are named after the German mathematician Ernst Schröder.

A large Schröder number $S_{n}$ describes the number of paths from the southwest corner $(0,0)$ of an $n \times n$ grid to the northeast corner $(n, n)$, using only single steps north, northeast, or east, that do not rise above the southwest-northeast diagonal. The first eleven large Schröder numbers $S_{n}$ for $0 \leq n \leq 10$ are

$$
\text { 1, 2, 6, 22, 90, 394, 1806, 8558, 41586, 206098, } 1037718 .
$$

The little Schröder numbers $s_{n}$ form an integer sequence that can be used to count the number of plane trees with a given set of leaves, the number of ways of inserting parentheses into a sequence, and the number of ways of dissecting a convex polygon into smaller polygons by inserting diagonals. The first eleven little Schröder numbers $s_{n}$ for $1 \leq n \leq 11$ are

$$
\text { 1, } \quad 1, \quad 3, \quad 11, \quad 45, \quad 197, \quad 903, \quad 4279, \quad 20793, \quad 103049, \quad 518859 .
$$

They are also called the small Schröder numbers or the Schröder-Hipparchus numbers, after Ernst Schröder and the ancient Greek mathematician Hipparchus who appears from evidence in Plutarch to have known of these numbers. They are also called the super-Catalan numbers, after Eugéne Charles Catalan, but different from a generalization of the Catalan numbers $[13,22,33]$.

1.2. Generating functions of the Schröder numbers. In [5, Theorem 8.5.7], it was proved that the large Schröder numbers $S_{n}$ have the generating function

$$
G(x)=\frac{1-x-\sqrt{x^{2}-6 x+1}}{2 x}=\sum_{n=0}^{\infty} S_{n} x^{n},
$$

which can also be rearranged as

$$
\mathcal{G}(x)=G(-x)=\frac{\sqrt{x^{2}+6 x+1}-1-x}{2 x}=\sum_{n=0}^{\infty}(-1)^{n} S_{n} x^{n} .
$$

In [5, Theorem 8.5.6], it was proved that the little Schröder numbers $s_{n}$ have the generating function

$$
g(x)=\frac{1+x-\sqrt{x^{2}-6 x+1}}{4}=\sum_{n=1}^{\infty} s_{n} x^{n} .
$$

For more information on the Schröder numbers $S_{n}$ and $s_{n}$, please refer to $[5,7]$ and plenty of references therein.

1.3. A Relation Between the Large and Little Schröder Numbers. From the generating functions (1.1) with (1.3), we can see that

$$
\sqrt{x^{2}-6 x+1}=1+x-4 \sum_{n=1}^{\infty} s_{n} x^{n}=1-x-2 \sum_{n=0}^{\infty} S_{n} x^{n+1},
$$


that is,

$$
1-2 \sum_{n=1}^{\infty} s_{n} x^{n-1}=1-2 \sum_{n=0}^{\infty} s_{n+1} x^{n}=-\sum_{n=0}^{\infty} S_{n} x^{n} .
$$

Accordingly, we acquire

$$
S_{n}=2 s_{n+1}, \quad n \in \mathbb{N} .
$$

See also [5, Corollary 8.5.8]. This relation shows that it is sufficient to analytically investigate the large Schröder numbers $S_{n}$.

1.4. Several Known Results. It is well known that there have been several simple formulas for the large Schröder numbers. For example, the large Schröder numbers can be computed by

$$
S_{n}=\sum_{k=0}^{n} \frac{1}{k+1}\left(\begin{array}{l}
n \\
k
\end{array}\right)\left(\begin{array}{c}
n+k \\
k
\end{array}\right)
$$

and satisfy the recursive relation

$$
S_{n}=S_{n-1}+\sum_{k=0}^{n-1} S_{k} S_{n-1-k}, \quad n \in \mathbb{N} .
$$

\section{Main Results for the Large Schröder Numbers}

Now we state our main results: several explicit formulas, a recursive formula, several integral representations, and some properties for the large Schröder numbers $S_{n}$.

2.1. Several Explicit Formulas for the Large Schröder Numbers. After studying the generating functions $G(x)$ and $\mathcal{G}(x)$, defined by (1.1) and (1.2), we obtain several explicit formulas for the large Schröder numbers $S_{n}$ by several approaches.

Theorem 2.1 ([25, Theorem 1]). For $n \in \mathbb{N}$, the large Schröder numbers $S_{n}$ can be computed by

$$
S_{n}=\frac{1}{2}\left(-\frac{1}{6}\right)^{n+1} \sum_{k=1}^{n+1}(-1)^{k} 9^{k} \frac{(2 k-3) ! !}{(2 k) !}\left(\begin{array}{c}
k \\
n-k+1
\end{array}\right)
$$

and

$$
\begin{aligned}
S_{n}= & \frac{1}{12} \frac{n !}{6^{n}} \sum_{k=0}^{n} \sum_{r+s=k} \sum_{\ell+m=n} \sum_{q=0}^{s} \sum_{j=0}^{n-r-1}(-1)^{s-q} 6^{2(r+j)}\left\langle\frac{1}{2}\right\rangle_{k}\left\langle\frac{1}{2}-k\right\rangle_{j} \\
& \times\left(\begin{array}{c}
\ell-1 \\
r-1
\end{array}\right)\left(\begin{array}{c}
s \\
q
\end{array}\right)\left(\begin{array}{c}
j \\
n-r-j+1
\end{array}\right)\left(\begin{array}{c}
m+2 q-1 \\
2 q-1
\end{array}\right) \frac{1}{r ! s ! j !},
\end{aligned}
$$

where $\left(\begin{array}{l}p \\ q\end{array}\right)=0$ for $q>p \geq 0$, the double factorial of negative odd integers $-2 n-1$ is defined by

$$
(-2 n-1) ! !=\frac{(-1)^{n}}{(2 n-1) ! !}=(-1)^{n} \frac{2^{n} n !}{(2 n) !}
$$


for $n=0,1, \ldots$, and $\langle x\rangle_{n}$ is the falling factorial

$$
\langle x\rangle_{n}=\prod_{k=0}^{n-1}(x-k)= \begin{cases}x(x-1) \cdots(x-n+1), & n \geq 1 \\ 1, & n=0\end{cases}
$$

Theorem 2.2 ([9, Theorem 4]). For $n \in \mathbb{N}$, the large Schröder numbers $S_{n}$ can be computed by

$$
S_{n}=-\frac{1}{2}(\sqrt{2}-1)^{ \pm 2(n+1)} \sum_{\ell=0}^{n+1} \frac{(2 \ell-3) ! !}{(2 \ell) ! !} \frac{[2(n-\ell+1)-3] ! !}{[2(n-\ell+1)] ! !}(\sqrt{2}+1)^{ \pm 4 \ell}
$$

and

$$
S_{n}=-\frac{1}{2}(\sqrt{2}+1)^{ \pm 2(n+1)} \sum_{\ell=0}^{n+1} \frac{(2 \ell-3) ! !}{(2 \ell) ! !} \frac{[2(n-\ell+1)-3] ! !}{[2(n-\ell+1)] ! !}(\sqrt{2}-1)^{ \pm 4 \ell}
$$

2.2. A Recursive Formula for the Large Schröder Numbers. Applying Leibniz's theorem to the equality (1.4), we can find the following recursive formula for the large Schröder numbers $S_{n}$.

Theorem 2.3 ([9, Theorem 5]). For $n \geq 0$, the large Schröder numbers $S_{n}$ satisfy the recursive formula

$$
S_{n+3}=3 S_{n+2}+\sum_{\ell=0}^{n} S_{\ell+1} S_{n-\ell+1}
$$

2.3. Integral Representations for the Large Schröder Numbers. In light of the Cauchy integral formula in the theory of complex functions, we can establish several integral representations for principal branches of the generating function $\mathcal{G}(z)=G(-z)$ on the cut complex plane $\mathbb{C} \backslash[-2 \sqrt{2}-3,2 \sqrt{2}-3]$ and, consequently, derive several integral representations for the large Schröder numbers $S_{n}$.

Theorem 2.4 ([23, Theorem 1.1]). The principal branch of the generating function

$$
\mathcal{G}(z)=\frac{\sqrt{z^{2}+6 z+1}-1-z}{2 z}
$$

on the cut complex plane $\mathbb{C} \backslash[-2 \sqrt{2}-3,2 \sqrt{2}-3]$ has the integral representations

$$
\begin{aligned}
& \mathcal{G}(z)=\frac{\sqrt{2}}{\pi} \int_{0}^{\infty} \varrho(s) e^{-(3-2 \sqrt{2}) s} \int_{1 / e}^{1} v^{z s-1} \mathrm{~d} v \mathrm{~d} s \\
& \mathcal{G}(z)=\frac{2 \sqrt{2}}{\pi} \int_{0}^{\infty} F(s) e^{-(3-2 \sqrt{2}) s} \int_{1 / e}^{1} v^{z s-1} \mathrm{~d} v \mathrm{~d} s
\end{aligned}
$$


and

$$
\begin{aligned}
\mathcal{G}(z) & =\frac{1}{2 \pi} \int_{0}^{\infty} s q(s) \int_{1 / e}^{1} v^{z s-1} \mathrm{~d} v \mathrm{~d} s \\
& =\frac{1}{2 \pi} \int_{3-2 \sqrt{2}}^{3+2 \sqrt{2}} \frac{\sqrt{(3+2 \sqrt{2}-u)(u-3+2 \sqrt{2})}}{u} \frac{1}{u+z} \mathrm{~d} u,
\end{aligned}
$$

where

$$
\begin{aligned}
& \varrho(s)=\int_{0}^{1 / 2}\left(\sqrt{\frac{1}{u}-1}-\frac{1}{\sqrt{1 / u-1}}\right)\left[e^{-4 \sqrt{2} s u}-e^{-4 \sqrt{2} s(1-u)}\right] \mathrm{d} u, \\
& F(s)=\int_{0}^{1}\left(\frac{1}{u}-1\right)^{1 / 2}\left[1-\frac{1}{2(1-u)}\right] e^{-4 \sqrt{2} s u} \mathrm{~d} u,
\end{aligned}
$$

and

$$
q(s)=\int_{3-2 \sqrt{2}}^{3+2 \sqrt{2}} \sqrt{(3+2 \sqrt{2}-u)(u-3+2 \sqrt{2})} e^{-s u} \mathrm{~d} u
$$

are positive. Consequently,

(a) the generating function $\mathcal{G}(t)$ is completely monotonic on $(2 \sqrt{2}-3, \infty)$ and a Stieltjes transform;

(b) the large Schröder numbers $S_{n}$ for $n \geq 0$ can be represented by the integral representations

$$
\begin{aligned}
S_{n}= & \frac{\sqrt{2}}{\pi} \frac{1}{(n+1) !} \int_{0}^{\infty} \varrho(s) e^{-(3-2 \sqrt{2}) s} s^{n} \mathrm{~d} s \\
= & \frac{\sqrt{2}}{\pi} \frac{1}{n+1} \int_{0}^{1 / 2}\left(\sqrt{\frac{1}{u}-1}-\frac{1}{\sqrt{1 / u-1}}\right) \\
& \times\left[\frac{1}{(3-2 \sqrt{2}+4 \sqrt{2} u)^{n+1}}-\frac{1}{(3+2 \sqrt{2}-4 \sqrt{2} u)^{n+1}}\right] \mathrm{d} u, \\
S_{n}= & \frac{2 \sqrt{2}}{\pi} \frac{1}{(n+1) !} \int_{0}^{\infty} F(s) e^{-(3-2 \sqrt{2}) s} s^{n} \mathrm{~d} s \\
= & -\frac{\sqrt{2}}{\pi} \frac{1}{n+1} \int_{0}^{1} \frac{2 u-1}{\sqrt{u(1-u)}} \frac{1}{(4 \sqrt{2} u+3-2 \sqrt{2})^{n+1}} \mathrm{~d} u,
\end{aligned}
$$

and

$$
\begin{aligned}
S_{n} & =\frac{1}{2 \pi} \frac{1}{(n+1) !} \int_{0}^{\infty} q(s) s^{n+1} \mathrm{~d} s \\
& =\frac{1}{2 \pi} \int_{3-2 \sqrt{2}}^{3+2 \sqrt{2}} \frac{\sqrt{(3+2 \sqrt{2}-u)(u-3+2 \sqrt{2})}}{u^{n+2}} \mathrm{~d} u .
\end{aligned}
$$


The second integral representations in (2.9) and (2.12) are simpler, more significant, and more useful.

2.4. Some Properties of the Large Schröder Numbers. Employing integral representations for the large Schröder numbers $S_{n}$, we can discover the following properties of the large Schröder numbers $S_{n}$.

Theorem 2.5 ([24, Theorem 1.1]). Let the Schröder function $S_{x}$ be defined by replacing $n$ by $x$ in (2.12). Then the function $(x+1) ! S_{x}$ for $x \geq 0$ is absolutely convex on $[0, \infty)$. Consequently, the sequence $\left\{(n+1) ! S_{n}\right\}_{n \geq 0}$ is absolutely convex and, particularly, convex.

The Schröder function $S_{x}$ is absolutely monotonic on $\left(-\frac{1}{2}, \infty\right)$ and completely monotonic on $\left(-\infty,-\frac{1}{2}\right)$. Consequently, the sequence $\left\{S_{n}\right\}_{n \geq 0}$ is absolutely monotonic and, particularly, convex.

Theorem 2.6 ([24, Theorem 1.2]). Let $x_{k} \in\{0\} \cup \mathbb{N}$ and $p_{k} \in \mathbb{N}$ such that $\sum_{\ell=1}^{n} \frac{1}{p_{\ell}}=1$ for $n \in \mathbb{N}$. Then

$$
S_{x_{0}}^{n-1} S_{\prod_{k=0}^{n} x_{k}} \geq \prod_{k=1}^{n} S_{x_{0}+x_{k}} \quad \text { and } \quad S_{\prod_{k=1}^{n} x_{k}} \leq S_{x_{1}}^{1 / p_{1}} \prod_{k=2}^{n} S_{x_{1}+p_{k} x_{k}}^{1 / p_{k}} .
$$

Theorem 2.7 ([24, Theorem 1.3]). Let $m \geq 1$ be a positive integer and let $\left|a_{i j}\right|_{m}$ denote a determinant of order $m$ with elements $a_{i j}$.

(a) If $a_{i}$ for $1 \leq i \leq m$ are non-negative integers, then

$$
\left|(-1)^{a_{i}+a_{j}}\left(a_{i}+a_{j}\right) ! S_{a_{i}+a_{j}}\right|_{m} \geq 0 \quad \text { and } \quad\left|\left(a_{i}+a_{j}\right) ! S_{a_{i}+a_{j}}\right|_{m} \geq 0 .
$$

(b) If $a=\left(a_{1}, a_{2}, \ldots, a_{n}\right)$ and $b=\left(b_{1}, b_{2}, \ldots, b_{n}\right)$ are non-increasing $n$-tuples of non-negative integers such that $\sum_{i=1}^{k} a_{i} \geq \sum_{i=1}^{k} b_{i}$ for $1 \leq k \leq n-1$ and $\sum_{i=1}^{n} a_{i}=\sum_{i=1}^{n} b_{i}$, then

$$
\prod_{i=1}^{n} \frac{S_{a_{i}}}{S_{b_{i}}} \geq \prod_{i=1}^{n} \frac{b_{i} !}{a_{i} !}
$$

Theorem 2.8 ([24, Theorem 1.4]). If $\ell \geq 0, n \geq k \geq m, 2 k \geq n$, and $2 m \geq n$, then

$$
\frac{S_{k+\ell} S_{n-k+\ell}}{S_{m+\ell} S_{n-m+\ell}} \geq \frac{(m+\ell) !(n-m+\ell) !}{(k+\ell) !(n-k+\ell) !} .
$$

Corollary 2.1 ([24, Corollary 1.1]). The sequence $n ! S_{n}$ for $n \geq 0$ is (logarithmically) convex.

Corollary 2.2 ([24, Corollary 1.2]). If $\ell \geq 0$ and $n \geq k \geq 0$, then

$$
\left[\frac{(n+\ell) ! S_{n+\ell}}{\ell ! S_{\ell}}\right]^{k} \geq\left[\frac{(k+\ell) ! S_{k+\ell}}{\ell ! S_{\ell}}\right]^{n}
$$


2.5. Relations Between the Large Schröder and Central Delannoy Numbers. Central Delannoy numbers $D(n)$ are the number of "king walks" from the $(0,0)$ corner of an $n \times n$ square to the upper right corner $(n, n)$. Central Delannoy numbers $D(n)$ have the generating function

$$
G(x)=\frac{1}{\sqrt{1-6 x+x^{2}}}=\sum_{k=0}^{\infty} D(k) x^{k}=1+3 x+13 x^{2}+63 x^{3}+\cdots .
$$

Central Delannoy numbers $D(n)$ and the large Schröder numbers $S_{n}$ can be represented each other in the form of determinants.

Theorem 2.9 ([24, Theorem 1.5]). For $k \in \mathbb{N}$, the large Schröder numbers $S_{k}$ and central Delannoy numbers $D(k)$ have relations

$$
S_{k}=\frac{(-1)^{k}}{2}\left|\begin{array}{ccccccc}
D(1) & 1 & 0 & \cdots & 0 & 0 & 0 \\
D(2) & D(1) & 1 & \cdots & 0 & 0 & 0 \\
D(3) & D(2) & D(1) & \cdots & 0 & 0 & 0 \\
\vdots & \vdots & \vdots & \ddots & \vdots & \vdots & \vdots \\
D(k-1) & D(k-2) & D(k-3) & \cdots & D(1) & 1 & 0 \\
D(k) & D(k-1) & D(k-2) & \cdots & D(2) & D(1) & 1 \\
D(k+1) & D(k) & D(k-1) & \cdots & D(3) & D(2) & D(1)
\end{array}\right|,
$$

$$
D(k)=2^{k}\left|\begin{array}{cccccccc}
\frac{3}{2} & -\frac{1}{2} & 0 & 0 & \cdots & 0 & 0 & 0 \\
S_{1} & \frac{3}{2} & -\frac{1}{2} & 0 & \cdots & 0 & 0 & 0 \\
S_{2} & S_{1} & \frac{3}{2} & -\frac{1}{2} & \cdots & 0 & 0 & 0 \\
\vdots & \vdots & \vdots & \vdots & \ddots & \vdots & \vdots & \vdots \\
S_{k-3} & S_{k-4} & S_{k-5} & S_{k-6} & \cdots & \frac{3}{2} & -\frac{1}{2} & 0 \\
S_{k-2} & S_{k-3} & S_{k-4} & S_{k-5} & \cdots & S_{1} & \frac{3}{2} & -\frac{1}{2} \\
S_{k-1} & S_{k-2} & S_{k-3} & S_{k-4} & \cdots & S_{2} & S_{1} & \frac{3}{2}
\end{array}\right|
$$

and

$$
D(k+1)=3 D(k)+2 \sum_{\ell=1}^{k} S_{\ell} D(k-\ell) .
$$

\section{Notions AND LEMmas}

In order to understand and prove our main results, we need the following notions and lemmas.

3.1. Notions. Recall from [17, Chapter XIII], [31, Chapter 1], and [37, Chapter IV] that an infinitely differentiable function $f$ is said to be completely monotonic on an interval $I$ if it satisfies $0 \leq(-1)^{k} f^{(k)}(t)<\infty$ on $I$ for all $k \geq 0$. It is known [37, p. 161, Theorem $12 \mathrm{~b}$ ] that a function $f$ is completely monotonic on $(0, \infty)$ if and only if it is a Laplace transform $f(t)=\int_{0}^{\infty} e^{-t s} \mathrm{~d} \mu(s)$ of a positive measure $\mu$ defined on $[0, \infty)$ such that the above integral converges on $(0, \infty)$. 
In $[2,21]$, it was defined implicitly and explicitly that an infinitely differentiable and positive function $f$ is said to be logarithmically completely monotonic on an interval $I$ if the inequality $0 \leq(-1)^{k}[\ln f(x)]^{(k)}<\infty$ holds on $I$ for all $k \in \mathbb{N}$. In [3, Theorem 1.1], [8, Theorem 4], and [21, Theorem 1], it was found and verified once again that a logarithmically completely monotonic function must be completely monotonic.

An infinitely differentiable and nonnegative function $f: I \rightarrow[0, \infty)$ is called a Bernstein function on an interval $I$ if $f^{\prime}(t)$ is completely monotonic on $I$. See [10, Definition 1.2]. Theorem 3.2 in [31] states that a function $f:(0, \infty) \rightarrow[0, \infty)$ is a Bernstein function if and only if it admits the Lévy-Khintchine representation

$$
f(x)=a+b x+\int_{0}^{\infty}\left(1-e^{-x t}\right) \mathrm{d} \mu(t)
$$

where $a, b \geq 0$ and $\mu$ is a Lévy measure on $(0, \infty)$, with $\int_{0}^{\infty} \min \{1, t\} \mathrm{d} \mu(t)<\infty$. The Lévy triplet $(a, b, \mu)$ determines $f$ uniquely and vice versa. In [6, pp. 161-162, Theorem 3] and [31, Proposition 5.25], it was proved that the reciprocal of a Bernstein function is logarithmically completely monotonic.

If $\mathrm{d} \mu(t)=m(t) \mathrm{d} t$ and $m(t)$ is a completely monotonic function on $(0, \infty)$, then the function $f$ in (3.1) is said to be a complete Bernstein function. See [31, Definition 6.1].

In [31, Definition 2.1], it was defined that a Stieltjes transform is a function $f$ : $(0, \infty) \rightarrow[0, \infty)$ which can be written in the form

$$
f(t)=\frac{a}{t}+b+\int_{0}^{\infty} \frac{1}{u+t} \mathrm{~d} \mu(u),
$$

where $a, b$ are nonnegative constants and $\mu$ is a nonnegative measure on $(0, \infty)$ such that $\int_{0}^{\infty} \frac{1}{1+s} \mathrm{~d} \mu(s)<\infty$. In [3, Theorem 1.2], it was proved that a positive Stieltjes transform must be a logarithmically completely monotonic function on $(0, \infty)$, but not conversely.

Recall from [37, p. 108, Definition 4] that a sequence $\mu_{n}$ for $n \geq 0$ is said to be completely monotonic if its elements are non-negative and its successive differences satisfy $(-1)^{k} \Delta^{k} \mu_{n} \geq 0$ for $n, k \geq 0$, where

$$
\Delta^{k} \mu_{n}=\sum_{m=0}^{k}(-1)^{m}\left(\begin{array}{c}
k \\
m
\end{array}\right) \mu_{n+k-m} .
$$

Theorem 4a in [37, p. 108] reads that a necessary and sufficient condition for the sequence $\mu_{n}$ for $n \geq 0$ to be completely monotonic is $\mu_{n}=\int_{0}^{1} t^{n} \mathrm{~d} \alpha(t)$ for $n \geq 0$, where $\alpha(t)$ is non-decreasing and bounded for $0 \leq t \leq 1$.

In [17, pp. 375-386], it was recited that, if $f \in C^{\infty}(a, b)$ and $f^{(2 k)}(x) \geq 0$ for $k \geq 0$ and $x \in[a, b]$, we say that $f$ is an absolutely convex function; if $f \in C^{\infty}(a, b)$ and $(-1)^{k} f^{(2 k)}(x) \geq 0$ for $k \geq 0$ and $x \in[a, b]$, then we say that $f$ is a completely convex function. Similarly, if elements of a sequence $\mu_{n}$ for $n \geq 0$ are non-negative and $(-1)^{k} \Delta^{2 k} \mu_{n} \geq 0$ for $n, k \geq 0$, then we say that the sequence $\mu_{n}$ for $n \geq 0$ is completely 
convex; if elements of a sequence $\mu_{n}$ for $n \geq 0$ are non-negative and $\Delta^{2 k} \mu_{n} \geq 0$ for $n, k \geq 0$, then we say that the sequence $\mu_{n}$ for $n \geq 0$ is absolutely convex.

For more information on the above notions, please refer to the monograph $[17$, Chapter XIII] and the papers [11, 12, 14,32].

3.2. Lemmas. In combinatorial mathematics, the Bell polynomials of the second kind $\mathrm{B}_{n, k}$ are defined by

$$
\mathrm{B}_{n, k}\left(x_{1}, x_{2}, \ldots, x_{n-k+1}\right)=\sum_{\substack{\ell_{i} \in\{0\} \cup \mathbb{N} \\ \sum_{i=1}^{n} i \ell_{i}=n \\ \sum_{i=1}^{n} \ell_{i}=k}} \frac{n !}{\prod_{i=1}^{n-k+1} \ell_{i} !} \prod_{i=1}^{n-k+1}\left(\frac{x_{i}}{i !}\right)^{\ell_{i}},
$$

for $n \geq k \geq 0$. See [7, p. 134, Theorem A]. In combinatorial analysis, the Faà di Bruno formula plays an important role and can be described by

$$
\frac{\mathrm{d}^{n}}{\mathrm{~d} t^{n}}[f \circ h(t)]=\sum_{k=0}^{n} f^{(k)}(h(t)) \mathrm{B}_{n, k}\left(h^{\prime}(t), h^{\prime \prime}(t), \ldots, h^{(n-k+1)}(t)\right)
$$

in terms of the Bell polynomials of the second kind $\mathrm{B}_{n, k}$. See $[7$, p. 139, Theorem C].

Lemma 3.1 ([7, p. 135]). For $n \geq k \geq 0$, we have

$$
\mathrm{B}_{n, k}\left(a b x_{1}, a b^{2} x_{2}, \ldots, a b^{n-k+1} x_{n-k+1}\right)=a^{k} b^{n} \mathrm{~B}_{n, k}\left(x_{1}, x_{2}, \ldots, x_{n-k+1}\right),
$$

where $a$ and $b$ are any complex numbers.

Lemma 3.2 ([30, Theorem 3.1] and [36, Lemma 2.5]). For $n \geq k \geq 0$, the Bell polynomials of the second kind $\mathrm{B}_{n, k}$ satisfy

$$
\mathrm{B}_{n, k}(x, 1,0, \ldots, 0)=\frac{(n-k) !}{2^{n-k}}\left(\begin{array}{l}
n \\
k
\end{array}\right)\left(\begin{array}{c}
k \\
n-k
\end{array}\right) x^{2 k-n} .
$$

Lemma 3.3 ([1, Example 2.6] and [7, p. 136, Eq. [3n]]). The Bell polynomials of the second kind $\mathrm{B}_{n, k}$ satisfy

$$
\begin{aligned}
& \mathrm{B}_{n, k}\left(x_{1}+y_{1}, x_{2}+y_{2}, \ldots, x_{n-k+1}+y_{n-k+1}\right) \\
= & \sum_{r+s=k} \sum_{\ell+m=n}\left(\begin{array}{l}
n \\
\ell
\end{array}\right) \mathrm{B}_{\ell, r}\left(x_{1}, x_{2}, \ldots, x_{\ell-r+1}\right) \mathrm{B}_{m, s}\left(y_{1}, y_{2}, \ldots, y_{m-s+1}\right) .
\end{aligned}
$$

Lemma 3.4 ([25, Lemma 6]). For $n \geq k \geq 0$, we have

$$
\mathrm{B}_{n, k}(1 !, 2 !, \ldots,(n-k+1) !)=\left(\begin{array}{l}
n \\
k
\end{array}\right)\left(\begin{array}{l}
n-1 \\
k-1
\end{array}\right)(n-k) !
$$

and

$$
\mathrm{B}_{n, k}(2 !, 3 !, \ldots,(n-k+2) !)=\frac{1}{k !} \sum_{\ell=0}^{k}(-1)^{k-\ell}\left(\begin{array}{c}
k \\
\ell
\end{array}\right) \frac{(n+2 \ell-1) !}{(2 \ell-1) !}
$$


Lemma 3.5 ([4, p. 40, Exercise 5)], [19, Section 2.2, p. 849], [20, p. 94], and [36, Lemma 2.1]). Let $u(x)$ and $v(x) \neq 0$ be two differentiable functions. Let $U_{(n+1) \times 1}(x)$ be an $(n+1) \times 1$ matrix whose elements $u_{k, 1}(x)=u^{(k-1)}(x)$ for $1 \leq k \leq n+1$, let $V_{(n+1) \times n}(x)$ be an $(n+1) \times n$ matrix whose elements

$$
v_{i, j}(x)= \begin{cases}\left(\begin{array}{l}
i-1 \\
j-1
\end{array}\right) v^{(i-j)}(x), & i-j \geq 0 \\
0, & i-j<0\end{cases}
$$

for $1 \leq i \leq n+1$ and $1 \leq j \leq n$, and let $\left|W_{(n+1) \times(n+1)}(x)\right|$ denote the determinant of the $(n+1) \times(n+1)$ matrix

$$
W_{(n+1) \times(n+1)}(x)=\left(U_{(n+1) \times 1}(x) \quad V_{(n+1) \times n}(x)\right) .
$$

Then the $n$th derivative of the ratio $\frac{u(x)}{v(x)}$ can be computed by

$$
\frac{\mathrm{d}^{n}}{\mathrm{~d} x^{n}}\left[\frac{u(x)}{v(x)}\right]=(-1)^{n} \frac{\left|W_{(n+1) \times(n+1)}(x)\right|}{v^{n+1}(x)} .
$$

Lemma 3.6 ([35, Theorem 2.1]). Let $M$ be a square matrix of order $n \times n$ and partitioned as

$$
M=\left(\begin{array}{cc}
A & B \\
C & D
\end{array}\right)
$$

Let $K^{-1}$ denote the inverse of an invertible matrix $K$.

(a) If $B$ is a $k \times k$ matrix and $C$ nonsingular, then the determinant of $M$ can be computed by

$$
|M|=(-1)^{(n+1) k}|C|\left|B-A C^{-1} D\right| .
$$

(b) If $A, B, C, D$ are respectively $p \times p, p \times q, q \times p$, and $q \times q$ matrices and if $D$ is invertible, then the determinant of $M$ can be computed by

$$
|M|=|D|\left|A-B D^{-1} C\right| \text {. }
$$

Lemma 3.7 ([29, Theorem 4.2]). For $a>b>0$ and $z \in \mathbb{C} \backslash[-a,-b]$, the principal branch of the geometric mean

$$
G_{a, b}(z)=\sqrt{(a+z)(b+z)}
$$

has the integral representation

$$
G_{a, b}(z)=G_{a, b}(0)+z+\frac{a-b}{2 \pi} \int_{0}^{\infty} \frac{\rho((a-b) s)}{s} e^{-b s}\left(1-e^{-z s}\right) \mathrm{d} s
$$

where $\rho(s)$ is defined by

$$
\rho(s)=\int_{0}^{1 / 2}\left(\sqrt{\frac{1}{u}-1}-\frac{1}{\sqrt{1 / u-1}}\right)\left[1-e^{-(1-2 u) s}\right] e^{-s u} \mathrm{~d} u .
$$


Lemma 3.8 ([28, Theorem 1.1]). For $\lambda \in(0,1)$ and $a>b>0$, the principal branch of the weighted geometric mean

$$
G_{a, b ; \lambda}(z)=(a+z)^{\lambda}(b+z)^{1-\lambda},
$$

for $\lambda \in(0,1)$ and $z \in \mathbb{C} \backslash[-a,-b]$ has the integral representation

$$
G_{a, b ; \lambda}(z)=a^{\lambda} b^{1-\lambda}+z+\frac{\sin (\lambda \pi)}{\pi}(a-b) \int_{0}^{\infty} \frac{F(\lambda,(a-b) s)}{s} e^{-b s}\left(1-e^{-z s}\right) \mathrm{d} s,
$$

where

$$
F(\lambda, s)=\int_{0}^{1}\left(\frac{1}{u}-1\right)^{\lambda}\left(1-\frac{\lambda}{1-u}\right) e^{-s u} \mathrm{~d} u
$$

is positive for all $(\lambda, s) \in(0,1) \times(0, \infty)$.

Lemma 3.9 ([27, Theorem 1.1]). Let $n \in \mathbb{N}$ and $\mathbf{a}=\left(a_{1}, a_{2}, \ldots, a_{n}\right)$ be a positive sequence, that is, $a_{k}>0$ for $1 \leq k \leq n$, and no any two are same. For $z \in$ $\mathbb{C} \backslash\left[-\max \left\{a_{k}, 1 \leq k \leq n\right\},-\min \left\{a_{k}, 1 \leq k \leq n\right\}\right]$ and $n \geq 2$, let $\mathbf{1}=(\overbrace{1,1, \ldots, 1}^{n})$ and

$$
G_{n}(\mathbf{a}+z \mathbf{1})=\left[\prod_{k=1}^{n}\left(a_{k}+z\right)\right]^{1 / n}
$$

Let $\sigma$ be a permutation of the set $\{1,2, \ldots, n\}$ such that $\sigma(\mathbf{a})=\left(a_{\sigma(1)}, a_{\sigma(2)}, \ldots, a_{\sigma(n)}\right)$ is a rearrangement of $\mathbf{a}$ in an ascending order $a_{\sigma(1)}<a_{\sigma(2)}<\cdots<a_{\sigma(n)}$. Then the principal branch of the geometric mean $G_{n}(\mathbf{a}+z \mathbf{1})$ has the Lévy-Khintchine representation

$$
G_{n}(\mathbf{a}+z \mathbf{1})=G_{n}(\mathbf{a})+z+\int_{0}^{\infty} Q_{n, \mathbf{a}}(u)\left(1-e^{-z u}\right) \mathrm{d} u
$$

where

$$
Q_{n, \mathbf{a}}(u)=\frac{1}{\pi} \sum_{\ell=1}^{n-1} \sin \frac{\ell \pi}{n} \int_{a_{\sigma(\ell)}}^{a_{\sigma(\ell+1)}}\left|\prod_{k=1}^{n}\left(a_{k}-t\right)\right|^{1 / n} e^{-u t} \mathrm{~d} t .
$$

Lemma 3.10 ([10, Theorem 2.2] and [26, Theorems 3.1 and 4.6]). Let $w_{k}>0$ and $\sum_{k=1}^{n} w_{k}=1$ for $1 \leq k \leq n$ and $n \geq 2$. If $a=\left(a_{1}, a_{2}, \ldots, a_{n}\right)$ is a positive and strictly increasing sequence, that is, $0<a_{1}<a_{2}<\cdots<a_{n}$, then the principal branch of the weighted geometric mean

$$
G_{w, n}(a+z)=\prod_{k=1}^{n}\left(a_{k}+z\right)^{w_{k}},
$$

for $z \in \mathbb{C} \backslash\left[-a_{n},-a_{1}\right]$ has the Lévy-Khintchine representation

$$
G_{w, n}(a+z)=G_{w, n}(a)+z+\int_{0}^{\infty} m_{a, w, n}(u)\left(1-e^{-z u}\right) \mathrm{d} u,
$$


where the density

$$
m_{a, w, n}(u)=\frac{1}{\pi} \sum_{\ell=1}^{n-1} \sin \left(\pi \sum_{j=1}^{\ell} w_{j}\right) \int_{a_{\ell}}^{a_{\ell+1}} \prod_{k=1}^{n}\left|a_{k}-t\right|^{w_{k}} e^{-u t} \mathrm{~d} t .
$$

Lemma 3.11 ([24, Lemma 2.2]). For $\alpha>1, \beta \in \mathbb{R}$, and $k \in \mathbb{N}$, the definite integral

$$
I(\alpha, \beta ; k)=\int_{1 / \alpha}^{\alpha} \sqrt{\left(u-\frac{1}{a}\right)(a-u)} \frac{\ln ^{2 k-1} u}{u^{\beta}} \mathrm{d} u \begin{cases}<0, & \beta>\frac{3}{2} \\ =0, & \beta=\frac{3}{2} \\ >0, & \beta<\frac{3}{2}\end{cases}
$$

Lemma 3.12 ([20, Lemma 2.4]). Let $f(t)=1+\sum_{k=1}^{\infty} a_{k} t^{k}$ and $g(t)=1+\sum_{k=1}^{\infty} b_{k} t^{k}$ be formal power series such that $f(t) g(t)=1$. Then

$$
b_{k}=(-1)^{k}\left|\begin{array}{cccccc}
a_{1} & 1 & 0 & 0 & \cdots & 0 \\
a_{2} & a_{1} & 1 & 0 & \cdots & 0 \\
a_{3} & a_{2} & a_{1} & 1 & \cdots & 0 \\
\vdots & \vdots & \vdots & \vdots & \ddots & \vdots \\
a_{k-1} & a_{k-2} & a_{k-3} & a_{k-4} & \cdots & 1 \\
a_{k} & a_{k-1} & a_{k-2} & a_{k-3} & \cdots & a_{1}
\end{array}\right| .
$$

4. Proofs of Main Results

We are now in a position to outline proofs of our main results.

4.1. Six proofs of the formula (2.1). The explicit formula (2.1) was proved by five approaches in [25].

4.1.1. First Proof of the Formula (2.1). The outline of the first proof is utilizing the expansion

$$
\mathcal{G}(x)=-\frac{1}{2}+\frac{1}{2} \sum_{k=1}^{\infty}\left\langle\frac{1}{2}\right\rangle_{k} \frac{1}{k !} x^{k-1}(x+6)^{k}, \quad|x(x+6)|<1,
$$

which can be derived from

$$
\sqrt{x^{2}+6 x+1}=\sum_{k=0}^{\infty}\left\langle\frac{1}{2}\right\rangle_{k} \frac{1}{k !}[x(x+6)]^{k}, \quad|x(x+6)|<1,
$$

and differentiating on both sides of (4.1).

4.1.2. Second Proof of the Formula (2.1). Making use of the formulas (3.2), (3.3), and (3.4) to compute the $m$ th derivative of $\sqrt{x^{2}+6 x+1}$ results in the formula (2.1).

4.1.3. Third Proof of the Formula (2.1). Applying Lemma 3.5 to the functions

$$
u(x)=\sqrt{x^{2}+6 x+1}-1-x
$$

and $v(x)=x$ and employing the formulas (3.8) and (3.9) by induction result in the formula (2.1). 
4.1.4. Fourth Proof of the Formula (2.1). Employing (3.9) to compute the determinant obtained in the third proof leads to the formula (2.1) once again.

4.1.5. Fifth Proof of the Formula (2.1). Combining the equation (1.2) with (4.2) finds

$$
\sum_{k=0}^{\infty}\left\langle\frac{1}{2}\right\rangle_{k} \frac{1}{k !}[x(x+6)]^{k}-1-x=2 \sum_{k=0}^{\infty}(-1)^{k} S_{k} x^{k+1} .
$$

The explicit formula (2.1) is thus proved once again.

4.1.6. Sixth Proof of the Formula (2.1). It suffices to show that the right-hand sides of the formulas (1.5) and (2.1) are equal, that is,

$$
\sum_{k=0}^{n} \frac{1}{k+1}\left(\begin{array}{l}
n \\
k
\end{array}\right)\left(\begin{array}{c}
n+k \\
k
\end{array}\right)=\frac{1}{2}\left(-\frac{1}{6}\right)^{n+1} \sum_{k=1}^{n+1}(-1)^{k} 9^{k} \frac{(2 k-3) ! !}{(2 k) !}\left(\begin{array}{c}
k \\
n-k+1
\end{array}\right),
$$

which can be carried out by Zeilberger's algorithm.

4.2. Proof of the Formula (2.2). The generating function (1.1) can be rearranged as

$$
G(x)=\frac{1}{2}\left(\frac{1}{x}-1-\sqrt{1-\frac{6}{x}+\frac{1}{x^{2}}}\right), \quad x>0 .
$$

Then, by virtue of the formulas (3.2) and (3.5), the $n$th derivative of $G(x)$ equals

$$
\begin{aligned}
2 G^{(n)}(x)= & \frac{(-1)^{n} n !}{x^{n+1}}-\sum_{k=0}^{n}\left\langle\frac{1}{2}\right\rangle_{k}\left(1-\frac{6}{x}+\frac{1}{x^{2}}\right)^{1 / 2-k} \\
& \times \sum_{r+s=k} \sum_{\ell+m=n}\left(\begin{array}{l}
n \\
\ell
\end{array}\right) \mathrm{B}_{\ell, r}\left(\frac{6}{x^{2}},-\frac{12}{x^{3}}, \ldots,(-1)^{\ell-r} \frac{6(\ell-r+1) !}{x^{\ell-r+2}}\right) \\
& \times \mathrm{B}_{m, s}\left(-\frac{2}{x^{3}}, \frac{6}{x^{4}}, \ldots,(-1)^{m-s+1} \frac{(m-s+2) !}{x^{m-s+3}}\right)
\end{aligned}
$$

for $n \in \mathbb{N}$. Further making use of the formulas (3.3), (3.6), and (3.7) reveals

$$
\begin{aligned}
2 G^{(n)}(x) \rightarrow & \frac{(-1)^{n+1}}{(n+1) !} \sum_{k=0}^{n}\left\langle\frac{1}{2}\right\rangle \sum_{r+s=k} \sum_{\ell+m=n}\left(\begin{array}{l}
n \\
\ell
\end{array}\right) 6^{r}(-1)^{r}\left(\begin{array}{l}
\ell \\
r
\end{array}\right)\left(\begin{array}{l}
\ell-1 \\
r-1
\end{array}\right) \frac{(\ell-r) !}{s !} \\
& \times \sum_{q=0}^{s}(-1)^{s-q}\left(\begin{array}{l}
s \\
q
\end{array}\right) \frac{(m+2 q-1) !}{(2 q-1) !}(k-s) !\left(\begin{array}{l}
n+1 \\
k-s
\end{array}\right) \\
& \times \lim _{x \rightarrow 0^{+}}\left[\left(x^{2}-6 x+1\right)^{1 / 2-k}\right]^{(n-k+s+1)}
\end{aligned}
$$


as $x \rightarrow 0^{+}$. By the formulas (3.2), (3.3), and (3.4), we have

$$
\begin{aligned}
& \lim _{x \rightarrow 0^{+}}\left[\left(x^{2}-6 x+1\right)^{1 / 2-k}\right]^{(n-k+s+1)} \\
= & \sum_{j=0}^{n-k+s-1}(-1)^{2 j-n+k-s-1}\left\langle\frac{1}{2}-k\right\rangle_{j} \\
& \times \frac{(n-k-j+s+1) !}{6^{n-k-2 j+s+1}}\left(\begin{array}{c}
n-k+s+1 \\
j
\end{array}\right)\left(\begin{array}{c}
j \\
n-k-j+s+1
\end{array}\right) .
\end{aligned}
$$

As a result, we can obtain the formula (2.2).

4.3. Proof of Theorem 2.2. From (1.2), it follows that

$$
\sqrt{x^{2}+6 x+1}=1+2 x+2 \sum_{n=2}^{\infty}(-1)^{n-1} S_{n-1} x^{n},
$$

which implies that, for $n \geq 2$,

$$
\begin{aligned}
2(-1)^{n-1} n ! S_{n-1} & =\lim _{x \rightarrow 0}(\sqrt{x+3+2 \sqrt{2}} \sqrt{x+3-2 \sqrt{2}})^{(n)} \\
& =\frac{(-1)^{n} n !}{(3-2 \sqrt{2})^{n}} \sum_{\ell=0}^{n} \frac{(2 \ell-3) ! !}{(2 \ell) ! !} \frac{[2(n-\ell)-3] ! !}{[2(n-\ell)] ! !}(17-12 \sqrt{2})^{\ell} .
\end{aligned}
$$

Therefore, we obtain

$$
S_{n-1}=-\frac{1}{2(3-2 \sqrt{2})^{n}} \sum_{\ell=0}^{n} \frac{(2 \ell-3) ! !}{(2 \ell) ! !} \frac{[2(n-\ell)-3] ! !}{[2(n-\ell)] ! !}(17-12 \sqrt{2})^{\ell}, \quad n \geq 2 .
$$

Similarly, we have

$$
S_{n-1}=-\frac{1}{2(3+2 \sqrt{2})^{n}} \sum_{\ell=0}^{n} \frac{(2 \ell-3) ! !}{(2 \ell) ! !} \frac{[2(n-\ell)-3] ! !}{[2(n-\ell)] ! !}(17+12 \sqrt{2})^{\ell}, \quad n \geq 2 .
$$

Making use of

$$
17 \pm 12 \sqrt{2}=(3 \pm 2 \sqrt{2})^{2}=(\sqrt{2}+1)^{ \pm 4}=(\sqrt{2}-1)^{\mp 4}
$$

in the above formulas yields the explicit formulas (2.3) and (2.4). The proof of Theorem 2.2 is complete.

4.4. Proof of Theorem 2.3. From (1.1), it follows that

$$
\sqrt{x^{2}-6 x+1}=1-3 x-2 \sum_{n=2}^{\infty} S_{n-1} x^{n} .
$$


Squaring on both sides of the above equation and comparing coefficients of $x^{n}$ for $n \geq 4$ yield

$$
3 S_{n-2}-S_{n-1}+\sum_{\ell=0}^{n-4} S_{\ell+1} S_{n-\ell-3}=0 .
$$

Replacing $n$ by $n+4$ in this equality and simplifying immediately lead to the recursive formula (2.5). The proof of Theorem 2.3 is thus complete.

4.5. Proof of Theorem 2.4. It is not difficult to write the function $\mathcal{G}(z)$ as

$$
\mathcal{G}(z)=\frac{\sqrt{(z+3-2 \sqrt{2})(z+3+2 \sqrt{2})}-\sqrt{(3-2 \sqrt{2})(3+2 \sqrt{2})}-z}{2 z} .
$$

Applying $x=3+2 \sqrt{2}$ and $y=3-2 \sqrt{2}$ to Lemma 3.7 yields the integral representation (2.7) immediately.

From the positivity of the functions $\varrho(s)=\rho(4 \sqrt{2} s)$ on $(0, \infty)$, it follows that

$$
(-1)^{k} \mathcal{G}^{(k)}(t)=\frac{\sqrt{2}}{\pi} \int_{0}^{\infty} \varrho(s) e^{-(3-2 \sqrt{2}) s} s^{k} \int_{1 / e}^{1}\left[(-1)^{k}(\ln v)^{k}\right] v^{t s-1} \mathrm{~d} v \mathrm{~d} s>0
$$

for all $k \geq 0$. Hence, the function $\mathcal{G}(t)$ is completely monotonic on $(2 \sqrt{2}-3, \infty)$.

From (1.2) and the relation

$$
S_{n}=(-1)^{n} \frac{1}{n !} \mathcal{G}^{(n)}(0), \quad n \geq 0,
$$

it is not difficult to see that the integral representation (2.10) is valid.

Substituting $x=3+2 \sqrt{2}, y=3-2 \sqrt{2}$, and $\lambda=\frac{1}{2}$ into Lemma 3.8 results in the integral representation (2.8) readily.

Since $F(s)>0$, as stated in Lemma 3.8, we have

$$
(-1)^{k} \mathcal{G}^{(k)}(t)=\frac{2 \sqrt{2}}{\pi} \int_{0}^{\infty} F(s) e^{-(3-2 \sqrt{2}) s} s^{k} \int_{1 / e}^{1}\left[(-1)^{k}(\ln v)^{k}\right] v^{t s-1} \mathrm{~d} v \mathrm{~d} s>0 .
$$

By definition, the complete monotonicity of the function $\mathcal{G}(t)$ is verified again.

The integral representation (2.11) is readily acquired by considering again the relation (4.4) and interchanging the order of integrations.

Setting $n=2, a_{1}=3-2 \sqrt{2}$, and $a_{2}=3+2 \sqrt{2}$ in Lemma 3.9, or letting $n=2$, $a_{1}=3-2 \sqrt{2}, a_{2}=3+2 \sqrt{2}$, and $w_{1}=w_{2}=\frac{1}{2}$ in Lemma 3.10 , leads to

$$
\mathcal{G}(z)=\frac{1}{2 z} \int_{0}^{\infty} Q_{2,(3-2 \sqrt{2}, 3+2 \sqrt{2})}(s)\left(1-e^{-z s}\right) \mathrm{d} s
$$

or

$$
\mathcal{G}(z)=\frac{1}{2 z} \int_{0}^{\infty} m_{(3-2 \sqrt{2}, 3+2 \sqrt{2}),(1 / 2,1 / 2), 2}(s)\left(1-e^{-z s}\right) \mathrm{d} s
$$


where

$$
\begin{aligned}
& Q_{2,(3-2 \sqrt{2}, 3+2 \sqrt{2})}(s)=m_{(3-2 \sqrt{2}, 3+2 \sqrt{2}),(1 / 2,1 / 2), 2}(s) \\
= & \frac{1}{\pi} \int_{3-2 \sqrt{2}}^{3+2 \sqrt{2}} \sqrt{(t-3+2 \sqrt{2})(3+2 \sqrt{2}-t)} e^{-s t} \mathrm{~d} t=\frac{q(s)}{\pi}
\end{aligned}
$$

is positive. Accordingly, we obtain the integral representation (2.9).

Since

$$
(-1)^{k} \mathcal{G}^{(k)}(t)=\frac{k !}{2 \pi} \int_{3-2 \sqrt{2}}^{3+2 \sqrt{2}} \frac{\sqrt{(3+2 \sqrt{2}-u)(u-3+2 \sqrt{2})}}{u(u+t)^{k+1}} \mathrm{~d} u>0
$$

for all $k \geq 0$, we recover the complete monotonicity of $\mathcal{G}(t)$ once again. On the other hand, from the relation (4.4), it is easy to see that the integral representation (2.12) is valid.

The function (2.6) can be rearranged as

$$
\sqrt{z^{2}+6 z+1}-1-z=2 \sum_{n=0}^{\infty}(-1)^{n} S_{n} z^{n+1}
$$

which implies that

$$
S_{n}=\frac{1}{2} \frac{(-1)^{n}}{(n+1) !} \lim _{z \rightarrow 0}[\sqrt{(z+3-2 \sqrt{2})(z+3+2 \sqrt{2})}]^{(n+1)},
$$

for $n \in \mathbb{N}$. As a result, utilizing Lemmas 3.7 to 3.10 and interchanging the order of integrations respectively, we obtain the integral representations (2.10) to (2.12) respectively once again. The proof of Theorem 2.4 is complete.

\subsection{Proof of Theorem 2.5. Let}

$$
f_{1}(x)=\int_{0}^{\infty} q(s) s^{x+1} \mathrm{~d} s, \quad x \geq 0 .
$$

It is apparent that

$$
f_{1}^{(k)}(x)=\int_{0}^{\infty} q(s)(\ln s)^{k} s^{x+1} \mathrm{~d} s, \quad x \geq 0 .
$$

This means that $f_{1}^{(2 k)}(x) \geq 0$ for $k \geq 0$ and $f_{1}(x)$ is absolutely convex on $[0, \infty)$. Consequently, by the first integral representation in $(2.12)$, the sequences $(n+1) ! S_{n}$ and $(n+1) ! s_{n+1}$ for $n \geq 0$ are absolutely convex.

It is obvious that $S_{x}$ for $x \geq 0$ is absolutely convex on $[0, \infty)$. Consequently, the sequences $S_{n}$ for $n \geq 0$ are absolutely convex. 
4.7. Proof of Theorem 2.6. In [15] and [17, pp. 369-370], it was obtained that, if $f$ is an absolutely monotonic function on $[0, \infty)$, then

$$
\left[f\left(x_{0}\right)\right]^{n-1} f\left(\prod_{k=0}^{n} x_{k}\right) \geq \prod_{k=1}^{n} f\left(x_{0}+x_{k}\right)
$$

and

$$
f\left(\prod_{k=1}^{n} x_{k}\right) \leq\left[f\left(x_{1}\right)\right]^{1 / p_{1}} \prod_{k=2}^{n}\left[f\left(x_{1}+p_{k} x_{k}\right)\right]^{1 / p_{k}},
$$

where $x_{\ell} \in[0, \infty)$ and $p_{\ell}>0$ such that $\sum_{\ell=1}^{n} \frac{1}{p_{\ell}}=1$. Applying $f(x)=S_{x}$ to these inequalities and taking $x_{\ell}, p_{\ell} \in\{0\} \cup \mathbb{N}$ lead to the inequalities in (2.13). The proof of Theorem 2.6 is complete.

4.8. Proof of Theorem 2.7. In [16] and [17, p. 367], it was obtained that, if $f$ is completely monotonic on $[0, \infty)$, then

$$
\left|f^{\left(a_{i}+a_{j}\right)}(x)\right|_{m} \geq 0 \quad \text { and } \quad\left|(-1)^{a_{i}+a_{j}} f^{\left(a_{i}+a_{j}\right)}(x)\right|_{m} \geq 0 .
$$

By Lemma 2.4, applying $f(x)=\mathcal{G}(x)$ in (2.9) to inequalities in (4.5) and taking the limit $x \rightarrow 0^{+}$give

$$
\lim _{x \rightarrow 0^{+}}\left|\mathcal{G}^{\left(a_{i}+a_{j}\right)}(x)\right|_{m}=\left|(-1)^{a_{i}+a_{j}}\left(a_{i}+a_{j}\right) ! S_{a_{i}+a_{j}}\right|_{m} \geq 0
$$

and

$$
\lim _{x \rightarrow 0^{+}}\left|(-1)^{a_{i}+a_{j}} \mathcal{G}^{\left(a_{i}+a_{j}\right)}(x)\right|_{m}=\left|(-1)^{a_{i}+a_{j}}(-1)^{a_{i}+a_{j}}\left(a_{i}+a_{j}\right) ! S_{a_{i}+a_{j}}\right|_{m} \geq 0 .
$$

The determinant inequalities in (2.14) follow.

In [17, p. 367, Theorem 2], it was stated that, if $f$ is a completely monotonic function on $[0, \infty)$, then

$$
\prod_{i=1}^{n}\left[(-1)^{a_{i}} f^{\left(a_{i}\right)}(x)\right] \geq \prod_{i=1}^{n}\left[(-1)^{b_{i}} f^{\left(b_{i}\right)}(x)\right] .
$$

By Lemma 2.4, replacing $f(x)$ by $\mathcal{G}(x)$ in (4.6) and taking the limit $x \rightarrow 0^{+}$give

$$
\lim _{x \rightarrow 0^{+}} \prod_{i=1}^{n}\left[(-1)^{a_{i}} \mathcal{G}^{\left(a_{i}\right)}(x)\right]=\prod_{i=1}^{n}\left(a_{i} ! S_{a_{i}}\right) \geq \lim _{x \rightarrow 0^{+}} \prod_{i=1}^{n}\left[(-1)^{b_{i}} \mathcal{G}^{\left(b_{i}\right)}(x)\right]=\prod_{i=1}^{n}\left(b_{i} ! S_{b_{i}}\right) .
$$

The product inequality (2.15) follows. The proof of Theorem 2.7 is complete.

4.9. Proof of Theorem 2.8. In [34, p. 397, Theorem D], it was recovered that if $f(x)$ is completely monotonic on $(0, \infty)$ and if $n \geq k \geq m, k \geq n-k$, and $m \geq n-m$, then

$$
(-1)^{n} f^{(k)}(x) f^{(n-k)}(x) \geq(-1)^{n} f^{(m)}(x) f^{(n-m)}(x) .
$$

By Lemma 2.4, replacing $f(x)$ by the function $(-1)^{\ell} \mathcal{G}^{(\ell)}(x)$ in the above inequality leads to

$$
(-1)^{n} \mathcal{G}^{(k+\ell)}(x) \mathcal{G}^{(n-k+\ell)}(x) \geq(-1)^{n} \mathcal{G}^{(m+\ell)}(x) \mathcal{G}^{(n-m+\ell)}(x) .
$$


Further taking $x \rightarrow 0^{+}$finds

$$
\begin{aligned}
& (-1)^{n}(-1)^{k+\ell}(k+\ell) ! S_{k+\ell}(-1)^{n-k+\ell}(n-k+\ell) ! S_{n-k+\ell} \\
\geq & (-1)^{n}(-1)^{m+\ell}(m+\ell) ! S_{m+\ell}(-1)^{n-m+\ell}(n-m+\ell) ! S_{n-m+\ell}
\end{aligned}
$$

which can be arranged as (2.16). The proof of Theorem 2.8 is complete.

4.10. Proof of Corollary 2.1. In [17, p. 369] and [18, p. 429, Remark], it was stated that, if $f(t)$ is a completely monotonic function such that $f^{(k)}(t) \neq 0$ for $k \geq 0$, then the sequence $\ln \left[(-1)^{k-1} f^{(k-1)}(t)\right]$ for $k \geq 1$ is convex. By Lemma 2.4, applying this result to the function $\mathcal{G}(x)$ figures out that the sequence

$$
\ln \left[(-1)^{k-1} \mathcal{G}^{(k-1)}(x)\right] \rightarrow \ln \left[(k-1) ! S_{k-1}\right], \quad x \rightarrow 0^{+}
$$

for $k \geq 1$ is convex. Hence, the sequence $n ! S_{n}$ for $n \geq 0$ is logarithmically convex.

Alternatively, letting $\ell \geq 1, n=2, a_{1}=\ell+2, a_{2}=\ell$, and $b_{1}=b_{2}=\ell+1$ in the inequality (2.15) leads to

$$
\left(\ell ! S_{\ell}\right)\left[(\ell+2) ! S_{\ell+2}\right] \geq[(\ell+1) !]^{2} S_{\ell+1}^{2},
$$

which means that the sequence $k ! S_{k}$ for $k \in \mathbb{N}$ is logarithmically convex. The proof of Corollary 2.1 is complete.

4.11. Proof of Corollary 2.2. This follows from taking

$$
a=(\overbrace{n+\ell, \ldots, n+\ell}^{k}, \overbrace{\ell, \ldots, \ell}^{n-k}) \text { and } \quad b=(k+\ell, k+\ell, \ldots, k+\ell)
$$

in the inequality (2.15). The proof of Corollary 2.2 is complete.

4.12. Proof of Theorem 2.9. Multiplying (4.3) with (2.17) yields

$$
\left(1+\sum_{k=1}^{\infty} D(k) t^{k}\right)\left(1-3 t-2 \sum_{k=2}^{\infty} S_{k-1} t^{k}\right)=1
$$

By virtue of Lemma 3.12, the forms (2.18) and (2.19) respectively follow immediately.

On the other hand, we can also expand the left-hand side of the equation (4.7) into a series

$$
\sum_{k=0}^{\infty}\left[D(k)-3 D(k-1)-2 \sum_{\ell=2}^{k} D(k-\ell) S_{\ell-1}\right] x^{k}=1 .
$$

The identity (2.20) is thus proved. The proof of Theorem 2.9 is complete. 
Acknowledgements. This paper is a revised version of a keynote talk, given on 21 April 2016, of the International Conference on Advances in Natural and Applied Sciences, April 21-23, 2016, Belek, Antalya, Turkey. The first author thanks Dr. Ahmet Ocak Akdemir at the Ağrı İbrahim Çeçen University in Turkey for his warming invitation, financial support, and generous hospitality for the first author to attend the International Conference.

The authors sincerely thank a lot the anonymous referees for their careful corrections to and valuable comments on the original version of this paper.

\section{REFERENCES}

[1] A. Aboud, J.-P. Bultel, A. Chouria, J.-G. Luque, O. Mallet, Bell polynomials in combinatorial Hopf algebras, arXiv (2014), Article ID 1402.2960v3.

[2] R. D. Atanassov and U. V. Tsoukrovski, Some properties of a class of logarithmically completely monotonic functions, C. R. Acad. Bulgare Sci. 41(2) (1988), 21-23.

[3] C. Berg, Integral representation of some functions related to the gamma function, Mediterr. J. Math. 1(4) (2004), 433-439.

[4] N. Bourbaki, Elements of Mathematics: Functions of a Real Variable: Elementary Theory, Translated from the 1976 French original by Philip Spain, Elements of Mathematics, SpringerVerlag, Berlin, 2004.

[5] R. A. Brualdi, Introductory Combinatorics, Fifth edition, Pearson Prentice Hall, Upper Saddle River, NJ, 2010.

[6] C.-P. Chen, F. Qi, and H. M. Srivastava, Some properties of functions related to the gamma and psi functions, Integral Transforms Spec. Funct. 21(2) (2010), 153-164.

[7] L. Comtet, Advanced Combinatorics: The Art of Finite and Infinite Expansions, Revised and Enlarged Edition, D. Reidel Publishing Co., Dordrecht and Boston, 1974.

[8] B.-N. Guo and F. Qi, A property of logarithmically absolutely monotonic functions and the logarithmically complete monotonicity of a power-exponential function, Politehn. Univ. Bucharest Sci. Bull. Ser. A Appl. Math. Phys. 72(2) (2010), 21-30.

[9] B.-N. Guo and F. Qi, Some explicit and recursive formulas of the large and little Schröder numbers, Arab J. Math. Sci. 23(1) (2017), in press, DOI 10.1016/j.ajmsc.2016.06.002.

[10] B.-N. Guo and F. Qi, On the degree of the weighted geometric mean as a complete Bernstein function, Afr. Mat. 26(7) (2015), 1253-1262.

[11] S. Guo, Some properties of completely monotonic sequences and related interpolation, Appl. Math. Comput. 219(10) (2013), 4958-4962.

[12] S. Guo, H. M. Srivastava, and N. Batir, A certain class of completely monotonic sequences, Adv. Difference Equ. 2013 (2013), Article ID 294.

[13] T. Koshy, Catalan Numbers with Applications, Oxford University Press, Oxford, 2009.

[14] D. S. Mitrinović and J. E. Pečarić, On completely monotonic sequences, Anz. Österreich. Akad. Wiss. Math.-Natur. Kl. 128 (1991), 63-67.

[15] D. S. Mitrinović and J. E. Pečarić, On some inequalities for monotone functions, Boll. Un. Mat. Ital. B (7) 5(2) (1991), 407-416.

[16] D. S. Mitrinović and J. E. Pečarić, On two-place completely monotonic functions, Anzeiger Öster. Akad. Wiss. Math.-Natturwiss. Kl. 126 (1989), 85-88.

[17] D. S. Mitrinović, J. E. Pečarić, and A. M. Fink, Classical and New Inequalities in Analysis, Kluwer Academic Publishers, Dordrecht-Boston-London, 1993.

[18] J. E. Pečarić, Remarks on some inequalities of A. M. Fink, J. Math. Anal. Appl. 104(2) (1984), 428-431. 
[19] F. Qi, Derivatives of tangent function and tangent numbers, Appl. Math. Comput. 268 (2015), $844-858$.

[20] F. Qi and R. J. Chapman, Two closed forms for the Bernoulli polynomials, J. Number Theory 159 (2016), 89-100.

[21] F. Qi and C.-P. Chen, A complete monotonicity property of the gamma function, J. Math. Anal. Appl. 296 (2004), 603-607.

[22] F. Qi, M. Mahmoud, X.-T. Shi, and F.-F. Liu, Some properties of the Catalan-Qi function related to the Catalan numbers, SpringerPlus 5 (2016), Article ID 1126.

[23] F. Qi, X.-T. Shi, and B.-N. Guo, Integral representations of the large and little Schröder numbers, ResearchGate Working Paper (2016), DOI 10.13140/RG.2.1.1988.3288.

[24] F. Qi, X.-T. Shi, and B.-N. Guo, Some properties of the Schröder numbers, Indian J. Pure Appl. Math. 47 (2016), in press.

[25] F. Qi, X.-T. Shi, and B.-N. Guo, Two explicit formulas of the Schröder numbers, Integers 16 (2016), Article ID A23.

[26] F. Qi, X.-J. Zhang, and W.-H. Li, An integral representation for the weighted geometric mean and its applications, Acta Math. Sin. (Engl. Ser.) 30(1) (2014), 61-68.

[27] F. Qi, X.-J. Zhang, and W.-H. Li, Lévy-Khintchine representation of the geometric mean of many positive numbers and applications, Math. Inequal. Appl. 17(2) (2014), 719-729.

[28] F. Qi, X.-J. Zhang, and W.-H. Li, Lévy-Khintchine representations of the weighted geometric mean and the logarithmic mean, Mediterr. J. Math. 11(2) (2014), 315-327.

[29] F. Qi, X.-J. Zhang, and W.-H. Li, The harmonic and geometric means are Bernstein functions, Bol. Soc. Mat. Mex. (3) 22 (2016), in press, DOI 10.1007/s40590-016-0085-y.

[30] F. Qi and M.-M. Zheng, Explicit expressions for a family of the Bell polynomials and applications, Appl. Math. Comput. 258 (2015), 597-607.

[31] R. L. Schilling, R. Song, and Z. Vondraček, Bernstein Functions - Theory and Applications, 2nd edition, de Gruyter Studies in Mathematics 37, Walter de Gruyter, Berlin, Germany, 2012.

[32] I. J. Schoenberg, On finite and infinite completely monotonic sequences, Bull. Amer. Math. Soc. 38(2) (1932), 72-76.

[33] R. P. Stanley, Catalan Numbers, Cambridge University Press, New York, 2015.

[34] H. van Haeringen, Completely monotonic and related functions, J. Math. Anal. Appl. 204(2) (1996), 389-408.

[35] W. Wanicharpichat, Explicit minimum polynomial, eigenvector and inverse formula of doubly Leslie matrix, J. Appl. Math. Inform. 33(3-4) (2015), 247-260.

[36] C.-F. Wei and F. Qi, Several closed expressions for the Euler numbers, J. Inequal. Appl. 2015 (2015), Article ID 219.

[37] D. V. Widder, The Laplace Transform, Princeton Mathematical Series 6, Princeton University Press, Princeton, N. J., 1941.

\author{
${ }^{1}$ Institute of Mathematics \\ Henan Polytechnic University \\ JiAOZUO City, 454010 \\ Henan Province \\ CHINA
}

\author{
${ }^{2}$ College of Mathematics \\ Inner Mongolia University for Nationalities \\ Tongliao City, 028043 \\ Inner Mongolia Autonomous Region \\ CHINA
}


${ }^{3}$ Department of Mathematics, College of Science

Tianjin Polytechnic University

Tianjin City, 300387

CHINA

E-mail address: qifeng618@gmail.com

${ }^{4}$ SChOOL OF MAThematics AND InFORMATICS

Henan Polytechnic University

JiAOZUO City, 454010

Henan Province

China

E-mail address: bai.ni.guo@hotmail.com 